\title{
Projet d'articles sur la prévention et la répression des crimes contre l'humanité 2019
}

Texte adopté par la Commission du droit international à sa soixante et onzième session, en 2019, et soumis à l'Assemblée générale dans le cadre de son rapport sur les travaux de ladite session (A/74/10, para. 54). Le rapport sera reproduit dans l'Annuaire de la Commission du droit international, 2019, vol. II(2).

Copyright (c) Nations Unies 2019

\section{Prévention et répression des crimes contre l'humanité}

Ayant à l'esprit que tout au long de l'histoire, des millions d'enfants, de femmes et d'hommes ont été victimes de crimes qui heurtent profondément la conscience humaine,

Reconnaissant que les crimes contre l'humanité menacent la paix, la sécurité et le bien-être du monde,

Rappelant les principes du droit international consacrés dans la Charte des Nations Unies,

Rappelant également que l'interdiction des crimes contre l'humanité constitue une norme impérative du droit international général (jus cogens),

Affirmant que les crimes contre l'humanité, qui sont parmi les crimes les plus graves touchant l'ensemble de la communauté internationale, doivent être prévenus en conformité avec le droit international,

Déterminés à mettre un terme à l'impunité des auteurs de ces crimes et à concourir ainsi à la prévention de nouveaux crimes,

Considérant la définition des crimes contre l'humanité énoncée à l'article 7 du Statut de Rome de la Cour pénale internationale,

Rappelant qu'il est du devoir de chaque État de soumettre à sa juridiction criminelle les crimes contre l'humanité,

Considérant les droits des victimes, témoins et autres personnes en relation avec des crimes contre l'humanité, ainsi que le droit des auteurs présumés des infractions à un traitement équitable,

Considérant également que puisque les crimes contre l'humanité ne sauraient rester impunis, la répression de tels crimes doit être effectivement assurée par des mesures prises dans le cadre national et par le renforcement de la coopération internationale, y compris en matière d'extradition et d'entraide judiciaire, 


\section{Article 1 Champ d'application}

Les présents projets d'article s'appliquent à la prévention et à la répression des crimes contre l'humanité.

\section{Article 2 Définition des crimes contre l'humanité}

1. Aux fins des présents projets d'article, on entend par " crime contre l'humanité » l'un quelconque des actes ci-après lorsqu'il est commis dans le cadre d'une attaque généralisée ou systématique lancée contre toute population civile et en connaissance de cette attaque:

a Meurtre;

b Extermination;

c Réduction en esclavage;

d Déportation ou transfert forcé de population;

e Emprisonnement ou autre forme de privation grave de liberté physique en violation des dispositions fondamentales du droit international;

f Torture;

g Viol, esclavage sexuel, prostitution forcée, grossesse forcée, stérilisation forcée ou toute autre forme de violence sexuelle de gravité comparable;

$\mathrm{h}$ Persécution de tout groupe ou de toute collectivité identifiable pour des motifs d'ordre politique, racial, national, ethnique, culturel, religieux ou sexiste, ou en fonction d'autres critères universellement reconnus comme inadmissibles en droit international, en corrélation avec tout acte visé dans le présent paragraphe;

i Disparition forcée de personnes;

j crime d'apartheid;

$\mathrm{k}$ autres actes inhumains de caractère analogue causant intentionnellement de grandes souffrances ou des atteintes graves à l'intégrité physique ou à la santé physique ou mentale.

2. Aux fins du paragraphe 1:

a par " attaque lancée contre une population civile ", on entend le comportement qui consiste en la commission multiple d'actes visés au paragraphe 1 à l'encontre d'une population civile quelconque, en application ou dans la poursuite de la politique d'un État ou d'une organisation ayant pour but une telle attaque;

b par « extermination », on entend notamment le fait d'imposer intentionnellement des conditions de vie, telles que la privation d'accès à la nourriture et aux médicaments, calculées pour entraîner la destruction d'une partie de la population;

c par "réduction en esclavage ", on entend le fait d'exercer sur une personne l'un quelconque ou l'ensemble des pouvoirs liés au droit de propriété, y compris dans le cadre de la traite des êtres humains, en particulier des femmes et des enfants;

d par "déportation ou transfert forcé de population ", on entend le fait de déplacer de force des personnes, en les expulsant ou par d'autres moyens 
coercitifs, de la région où elles se trouvent légalement, sans motifs admis en droit international;

e par " torture ", on entend le fait d'infliger intentionnellement une douleur ou des souffrances aiguës, physiques ou mentales, à une personne se trouvant sous sa garde ou sous son contrôle; l'acception de ce terme ne s'étend pas à la douleur ou aux souffrances résultant uniquement de sanctions légales, inhérentes à ces sanctions ou occasionnées par elles;

f par "grossesse forcée ", on entend la détention illégale d'une femme mise enceinte de force, dans l'intention de modifier la composition ethnique d'une population ou de commettre d'autres violations graves du droit international. Cette définition ne peut en aucune manière s'interpréter comme ayant une incidence sur les lois nationales relatives à la grossesse;

g par " persécution ", on entend le déni intentionnel et grave de droits fondamentaux en violation du droit international, pour des motifs liés à l'identité du groupe ou de la collectivité qui en fait l'objet;

h par " crime d'apartheid ", on entend des actes inhumains analogues à ceux que vise le paragraphe 1 , commis dans le cadre d'un régime institutionnalisé d'oppression systématique et de domination d'un groupe racial sur tout autre groupe racial ou tous autres groupes raciaux et dans l'intention de maintenir ce régime;

i par " disparition forcée de personnes ", on entend les cas où des personnes sont arrêtées, détenues ou enlevées par un État ou une organisation politique ou avec l'autorisation, l'appui ou l'assentiment de cet État ou de cette organisation, qui refuse ensuite d'admettre que ces personnes sont privées de liberté ou de révéler le sort qui leur est réservé ou l'endroit où elles se trouvent, dans l'intention de les soustraire à la protection de la loi pendant une période prolongée.

3. Ce projet d'article est sans préjudice de toute définition plus large prévue par tout instrument international, par le droit international coutumier ou par loi nationale.

\section{Article 3 Obligations générales}

1. Tout État a l'obligation de ne pas se livrer à des actes constitutifs de crimes contre l'humanité.

2. Les États s'engagent à prévenir et à punir les crimes contre l'humanité, qui sont des crimes au regard du droit international, qu'ils soient ou non commis en temps de conflit armé.

3. Aucune circonstance exceptionnelle, quelle qu'elle soit, qu'il s'agisse, entre autres, de conflit armé, d'instabilité politique intérieure ou d'un autre état d'exception, ne peut être invoquée pour justifier les crimes contre l'humanité.

\section{Article 4 Obligation de prévention}

Tout État s'engage à prévenir les crimes contre l'humanité, en conformité avec le droit international, au moyen de: 
a Mesures législatives, administratives, judiciaires et autres mesures efficaces de prévention dans tout territoire sous sa juridiction; et

b La coopération avec les autres États, les organisations intergouvernementales pertinentes et, selon qu'il convient, d'autres organisations.

\section{Article 5 Non-refoulement}

1. Aucun État n'expulse, ne refoule, ne remet ni n'extrade une personne vers un autre État s'il y a des motifs sérieux de croire qu'elle risque d'être victime d'un crime contre l'humanité.

2. Pour déterminer s'il y a de tels motifs, les autorités compétentes tiennent compte de toutes les considérations pertinentes, y compris, le cas échéant, de l'existence, dans l'État intéressé, d'un ensemble de violations systématiques graves, flagrantes ou massives des droits de l'homme ou de violations graves du droit international humanitaire.

\section{Article 6 Incrimination en droit interne}

1. Tout État prend les mesures nécessaires pour que les crimes contre l'humanité constituent des infractions au regard de son droit pénal.

2. Tout État prend les mesures nécessaires pour que les actes suivants constituent des infractions au regard de son droit pénal:

a Le fait de commettre un crime contre l'humanité;

b Le fait de tenter de commettre un tel crime; et

c Le fait d'ordonner, solliciter, encourager, apporter son aide, son concours ou toute autre forme d'assistance à la commission ou à la tentative de commission d'un tel crime.

3. Tout État prend également les mesures nécessaires pour que les chefs militaires et autres supérieurs hiérarchiques soient pénalement responsables des crimes contre l'humanité commis par leurs subordonnés s'ils savaient, ou avaient des raisons de savoir, que ces subordonnés s'apprêtaient à commettre ou commettaient de tels crimes et s'ils n'ont pas pris toutes les mesures nécessaires et raisonnables qui étaient en leur pouvoir pour en empêcher l'exécution ou, si ces crimes avaient déjà été commis, pour punir les responsables.

4. Tout État prend les mesures nécessaires pour que, au regard de son droit pénal, le fait qu'une infraction visée dans le présent projet d'article a été commise sur les ordres d'un gouvernement ou d'un supérieur, militaire ou civil, ne constitue pas un motif d'exonération de la responsabilité pénale d'un subordonné.

5. Tout État prend les mesures nécessaires pour que, au regard de son droit pénal, le fait qu'une infraction visée dans le présent projet d'article a été commise par une personne occupant une position officielle ne constitue pas un motif d'exonération de la responsabilité pénale.

6. Tout État prend les mesures nécessaires pour que, au regard de son droit pénal, les infractions visées dans le présent projet d'article ne se prescrivent pas. 
7. Tout État prend les mesures nécessaires pour que, au regard de son droit pénal, les infractions visées dans le présent projet d'article soient passibles de peines appropriées qui prennent en compte leur gravité.

8. Sous réserve des dispositions de son droit interne, tout État prend, s'il y a lieu, les mesures qui s'imposent, afin d'établir la responsabilité des personnes morales pour les infractions visées dans le présent projet d'article. Selon les principes juridiques de l'État, cette responsabilité peut être pénale, civile ou administrative.

\section{Article 7 Établissement de la compétence nationale}

1. Tout État prend les mesures nécessaires pour établir sa compétence aux fins de connaître des infractions couvertes par les présents projets d'article dans les cas suivants:

a Quand l'infraction a été commise sur tout territoire sous sa juridiction ou à bord d'un navire ou d'un aéronef immatriculé dans cet État;

b Quand l'auteur présumé de l'infraction est un ressortissant dudit État ou, si cet État le juge approprié, un apatride qui réside habituellement sur son territoire;

c Quand la victime est un ressortissant dudit État si cet État le juge approprié.

2. Tout État prend également les mesures nécessaires pour établir sa compétence aux fins de connaître des infractions couvertes par les présents projets d'article dans les cas où l'auteur présumé de l'infraction se trouve sur tout territoire sous sa juridiction et où il ne l'extrade ou ne le remet pas conformément aux présents projets d'article.

3. Les présents projets d'article n'excluent l'exercice d'aucune compétence pénale établie par un État conformément à son droit interne.

\section{Article 8 Enquête}

Tout État veille à ce que les autorités compétentes procèdent à une enquête rapide, approfondie et impartiale chaque fois qu'il y a des motifs raisonnables de croire que des actes constitutifs de crimes contre l'humanité ont été commis ou sont en train d'être commis sur tout territoire sous sa juridiction.

\section{Article 9 Mesures préliminaires lorsque l'auteur présumé de l'infraction se trouve sur le territoire}

1. S’il estime que les circonstances le justifient, après avoir examiné les renseignements dont il dispose, tout État sur le territoire sous la juridiction duquel se trouve une personne soupçonnée d'avoir commis une infraction couverte par les présents projets d'article assure la détention de cette personne ou prend toutes autres mesures juridiques nécessaires pour assurer sa présence. Cette détention et ces mesures doivent être conformes à la législation dudit État; elles ne peuvent être maintenues que pendant le délai nécessaire à l'engagement de poursuites pénales ou d'une procédure d'extradition ou de remise. 
2. Ledit État procède immédiatement à une enquête préliminaire en vue d'établir les faits.

3. Lorsqu'un État a mis une personne en détention préventive, conformément aux dispositions du présent projet d'article, il avise immédiatement de cette détention et des circonstances qui la justifient les États visés au paragraphe $1 \mathrm{du}$ projet d'article 7. L'État qui procède à l'enquête préliminaire visée au paragraphe 2 du présent projet d'article en communique rapidement les conclusions auxdits États, selon qu'il convient, et leur indique s'il entend exercer sa compétence.

\section{Article 10 Aut dedere aut judicare}

L'État sur le territoire sous la juridiction duquel l'auteur présumé de l'infraction se trouve, s'il n'extrade pas ce dernier vers un autre État ou ne le remet pas à une juridiction pénale internationale compétente, soumet l'affaire à ses autorités compétentes pour l'exercice de l'action pénale. Ces autorités prennent leur décision dans les mêmes conditions que pour toute autre infraction ayant un caractère grave en vertu du droit de cet État.

\section{Article 11 Traitement équitable de l'auteur présumé de l'infraction}

1. Toute personne objet de mesures à raison de l'une des infractions couvertes par les présents projets d'article jouit de la garantie à tous les stades de la procédure d'un traitement équitable, y compris d'un procès équitable, et de la pleine protection des droits qu'elle tire du droit interne et du droit international applicables, y compris le droit des droits de l'homme et le droit international humanitaire.

2. Toute personne qui est incarcérée, en état de détention préventive ou toute autre forme de détention dans un État autre que son État de nationalité est en droit:

a De communiquer sans retard avec le plus proche représentant qualifié de l'État ou des États dont elle a la nationalité ou qui est autrement habilité à protéger les droits de ladite personne ou, s'il s'agit d'une personne apatride, de l'État qui est disposé, sur la demande de cette personne, à protéger ses droits;

b De recevoir la visite d'un représentant de cet État ou de ces États; et

c D'être informée sans retard des droits que lui confère le présent paragraphe.

3. Les droits visés au paragraphe 2 s'exercent dans le cadre des lois et règlements de l'État sur le territoire sous la juridiction duquel se trouve la personne, étant entendu que ces lois et règlements doivent permettre la pleine réalisation des fins pour lesquelles les droits sont accordés en vertu du paragraphe 2 .

\section{Article 12 Victimes, témoins et autres personnes}

1. Chaque État prend les mesures nécessaires pour:

a Assurer à quiconque allègue que des actes constitutifs de crimes contre l'humanité ont été commis ou sont en train d'être commis le droit de porter plainte devant les autorités compétentes; et 
b Veiller à ce que les plaignants, les victimes, les témoins, ainsi que leurs proches et représentants, et toute autre personne participant à une enquête, à des poursuites ou à une procédure d'extradition ou une autre procédure relevant du champ d'application des présents projets d'article soient protégés contre les mauvais traitements ou intimidations en raison de toute plainte déposée, de toute communication d'informations, de tout témoignage ou de toute déposition faite. Les mesures de protection sont sans préjudice des droits de l'auteur présumé de l'infraction visés au projet d'article 11.

2. Chaque État fait en sorte, en conformité avec son droit interne, que les avis et préoccupations des victimes d'un crime contre l'humanité soient présentés et pris en compte aux stades appropriés de la procédure pénale engagée contre les auteurs présumés d'infractions, d'une manière qui ne porte pas préjudice aux droits visés au projet d'article 11 .

3. Chaque État prend les mesures qui s'imposent pour que son droit interne garantisse aux victimes d'un crime contre l'humanité commis sous la forme de faits attribuables à l'État en vertu du droit international ou commis sur tout territoire sous sa juridiction, le droit d'obtenir réparation des dommages matériels et moraux subis, à titre individuel ou collectif, consistant, le cas échéant, en une ou plusieurs des formes suivantes ou tout autre forme: restitution; indemnisation; satisfaction; réadaptation; cessation et garanties de non-répétition.

\section{Article 13 Extradition}

1. Le présent projet d'article s'applique aux infractions couvertes par les présents projets d'article, lorsqu'un État demande l'extradition d'une personne qui se trouve sur le territoire relevant de la juridiction d'un autre État.

2. Chacune des infractions couvertes par les présents projets d'article est de plein droit incluse dans tout traité d'extradition en vigueur entre les États en tant qu'infraction dont l'auteur peut être extradé. Les États s'engagent à inclure ces infractions en tant qu'infractions dont l'auteur peut être extradé dans tout traité d'extradition qu'ils concluront entre eux.

3. Pour les besoins de l'extradition entre États, les infractions couvertes par les présents projets d'article ne sont pas considérées comme des infractions politiques, des infractions connexes à des infractions politiques ou des infractions inspirées par des mobiles politiques. En conséquence, une demande d'extradition fondée sur une telle infraction ne peut être refusée pour ce seul motif.

4. Si un État qui subordonne l'extradition à l'existence d'un traité est saisi d'une demande d'extradition par un autre État avec lequel il n'est pas lié par un traité d'extradition, il peut considérer les présents projets d'article comme constituant la base juridique de l'extradition pour toute infraction couverte par les présents projets d'article.

5. Un État qui subordonne l'extradition à l'existence d'un traité, pour toute infraction couverte par les présents projets d'article:

a Informe le Secrétaire général de l'Organisation des Nations Unies s'il considère les présents projets d'article comme la base légale pour coopérer en matière d'extradition avec d'autres États; et 
b S'il ne considère pas les présents projets d'article comme la base légale pour coopérer en matière d'extradition, s'efforce, s'il y a lieu, de conclure des traités d'extradition avec d'autres États afin d'appliquer le présent projet d'article.

6. Les États qui ne subordonnent pas l'extradition à l'existence d'un traité reconnaissent entre eux, aux infractions couvertes par les présents projets d'article, le caractère d'infraction dont l'auteur peut être extradé.

7. L'extradition est subordonnée aux conditions prévues par le droit interne de l'État requis ou par les traités d'extradition applicables, y compris aux motifs pour lesquels l'État requis peut refuser l'extradition.

8. L'État requérant et l'État requis s'efforcent, sous réserve de leur droit interne, d'accélérer les procédures d'extradition et de simplifier les exigences en matière de preuve y relatives.

9. Si nécessaire, les infractions couvertes par les présents projets d'article sont réputées, aux fins de l'extradition entre États, avoir été commises tant au lieu de leur perpétration que sur le territoire des États ayant établi leur compétence conformément au paragraphe 1 du projet d'article 7 .

10. Si l'extradition, demandée aux fins d'exécution d'une peine, est refusée parce que la personne faisant l'objet de cette demande est un ressortissant de l'État requis, celui-ci, si son droit interne le lui permet, en conformité avec les prescriptions de ce droit et à la demande de l'État requérant, envisage de faire exécuter lui-même la peine prononcée, ou le reliquat de cette peine, conformément au droit interne de l'État requérant.

11. Aucune disposition des présents projets d'article ne doit être interprétée comme faisant obligation à l'État requis d'extrader s'il a de sérieuses raisons de penser que la demande a été présentée aux fins de poursuivre ou de punir une personne en raison de son sexe, de sa race, de sa religion, de sa nationalité, de son origine ethnique, de sa culture, de son appartenance à un certain groupe social, de ses opinions politiques, ou en fonction d'autres critères universellement reconnus comme inadmissibles en droit international, ou que donner suite à cette demande causerait un préjudice à cette personne pour l'une quelconque de ces raisons.

12. L'État requis prend dûment en considération la demande de l'État sur le territoire sous la juridiction duquel l'infraction présumée s'est produite.

13. Avant de refuser l'extradition, l'État requis consulte, selon qu'il convient, l'État requérant afin de lui donner toute possibilité de présenter ses opinions et de fournir des informations à l'appui de ses allégations.

\section{Article 14 Entraide judiciaire}

1. Les États s'accordent mutuellement l'entraide judiciaire la plus large possible lors des enquêtes, poursuites et procédures judiciaires concernant les infractions couvertes par les présents projets d'article conformément au présent projet d'article.

2. Concernant les infractions dont une personne morale peut être tenue responsable dans l'État requérant, conformément au paragraphe 8 du projet d'article 6, l'entraide judiciaire la plus large possible est accordée, autant que les lois, 
traités, accords et arrangements pertinents de l'État requis le permettent, lors des enquêtes, poursuites et procédures judiciaires et autres.

3. L'entraide judiciaire qui est accordée en application du présent projet d'article peut être demandée aux fins suivantes:

a Identifier et localiser les auteurs présumés de l'infraction et, le cas échéant, les victimes, témoins ou autres personnes;

b Recueillir des témoignages ou des dépositions, y compris par vidéoconférence;

c Signifier des actes judiciaires;

d Effectuer des perquisitions et des saisies;

e Examiner des objets et visiter des lieux, y compris le fait d'obtenir des preuves médico-légales;

f Fournir des informations, des pièces à conviction et des estimations d'experts;

g Fournir des originaux ou des copies certifiées conformes de documents et dossiers pertinents;

$\mathrm{h}$ Identifier, localiser ou geler des produits du crime, des biens, des instruments ou d'autres choses afin de recueillir des éléments de preuve ou à toutes autres fins;

i Faciliter la comparution volontaire de personnes dans l'État requérant; ou

j Fournir tout autre type d'assistance compatible avec le droit interne de l'État requis.

4. Les États ne peuvent invoquer le secret bancaire pour refuser l'entraide judiciaire prévue au présent projet d'article.

5. Les États envisagent, s'il y a lieu, la possibilité de conclure des accords ou des arrangements bilatéraux ou multilatéraux qui servent les objectifs du présent projet d'article, mettent en pratique ses dispositions ou les renforcent.

6. Sans préjudice de son droit interne, les autorités compétentes d'un État peuvent, sans demande préalable, communiquer des informations concernant des crimes contre l'humanité à une autorité compétente d'un autre État, si elles pensent que ces informations pourraient aider celle-ci à entreprendre ou à mener à bien des enquêtes, poursuites et procédures judiciaires, ou amener ce dernier État à formuler une demande en vertu des présents projets d'article.

7. Les dispositions du présent projet d'article n'affectent en rien les obligations découlant de tout autre traité bilatéral ou multilatéral régissant ou devant régir, entièrement ou partiellement, l'entraide judiciaire entre les États en question.

8. Le projet d'annexe aux présents projets d'article est applicable aux demandes faites conformément au présent projet d'article si les États en question ne sont pas liés par un traité d'entraide judiciaire. Si lesdits États sont liés par un tel traité, les dispositions correspondantes de ce traité sont applicables, à moins que les États ne conviennent d'appliquer à leur place les dispositions du projet d'annexe. Les États sont encouragés à appliquer le projet d'annexe s'il facilite la coopération. 
9. Les États envisagent, selon qu'il convient, de conclure des accords ou arrangements avec des mécanismes internationaux établis par les Nations Unies ou par d'autres organisations internationales et ayant pour mandat de recueillir des éléments de preuve concernant les crimes contre l'humanité.

\section{Article 15 Règlement des différends}

1. Les États s'efforcent de régler les différends concernant l'interprétation ou l'application des présents projets d'article par voie de négociation.

2. Tout différend entre deux ou plusieurs États touchant l'interprétation ou l'application des présents projets d'article qui n'aura pas été réglé par voie de négociation sera porté, à la requête de toute partie au différend, devant la Cour internationale de Justice, à moins que ces États ne conviennent de soumettre le différend à l'arbitrage.

3. Chaque État peut déclarer qu'il ne s'estime pas lié par le paragraphe $2 \mathrm{du}$ présent projet d'article. Les autres États ne sont pas liés par le paragraphe $2 \mathrm{du}$ présent projet d'article envers tout État ayant fait une telle déclaration.

4. Tout État ayant fait une déclaration en application du paragraphe 3 du présent projet d'article peut la retirer à tout moment.

\section{Annexe}

1. Ce projet d'annexe s'applique en conformité avec le paragraphe 8 du projet d'article 14.

\section{Désignation d'une autorité centrale}

2. Chaque État désigne une autorité centrale qui a la responsabilité et le pouvoir de recevoir les demandes d'entraide judiciaire et soit de les exécuter, soit de les transmettre aux autorités compétentes pour exécution. Si un État a une région ou un territoire spécial doté d'un système d'entraide judiciaire différent, il peut désigner une autorité centrale distincte qui aura la même fonction pour ladite région ou ledit territoire. Les autorités centrales assurent l'exécution ou la transmission rapide et en bonne et due forme des demandes reçues. Si l'autorité centrale transmet la demande à une autorité compétente pour exécution, elle encourage l'exécution rapide et en bonne et due forme de la demande par l'autorité compétente. L'autorité centrale désignée à cette fin fait l'objet d'une notification par chaque État adressée au Secrétaire général de l'Organisation des Nations Unies. Les demandes d'entraide judiciaire et toute communication y relative sont transmises aux autorités centrales désignées par les États. La présente disposition s'entend sans préjudice du droit de tout État d'exiger que ces demandes et communications lui soient adressées par la voie diplomatique et, en cas d'urgence, si les États en conviennent, par l'intermédiaire de l'Organisation internationale de police criminelle, si cela est possible. 


\section{Procédures de présentation des demandes}

3. Les demandes sont adressées par écrit ou, si possible, par tout autre moyen pouvant produire un document écrit, dans une langue acceptable pour l'État requis, dans des conditions permettant audit État d'en établir l'authenticité. La ou les langues acceptables pour chaque État sont notifiées au Secrétaire général de l'Organisation des Nations Unies par chacun de ces États. En cas d'urgence et si les États en conviennent, les demandes peuvent être faites oralement mais doivent être confirmées sans délai par écrit.

4. Une demande d'entraide judiciaire doit contenir les renseignements suivants:

a La désignation de l'autorité dont émane la demande;

b L'objet et la nature de l'enquête, des poursuites ou de la procédure judiciaire auxquelles se rapporte la demande, ainsi que le nom et les fonctions de l'autorité qui en est chargée;

c Un résumé des faits pertinents, sauf pour les demandes adressées aux fins de la signification d'actes judiciaires;

d Une description de l'assistance requise et le détail de toute procédure particulière que l'État requérant souhaite voir appliquée;

e Si possible, l'identité, l'adresse et la nationalité de toute personne visée; et

f Le but dans lequel le témoignage, les informations ou les mesures sont demandés.

5. L'État requis peut demander un complément d'information lorsque cela apparaît nécessaire pour exécuter la demande conformément à son droit interne ou lorsque cela peut en faciliter l'exécution.

\section{Réponse de l'État requis}

6. Toute demande est exécutée conformément au droit interne de l'État requis et, dans la mesure où cela ne contrevient pas au droit interne de l'État requis et lorsque cela est possible, conformément aux procédures spécifiées dans la demande.

7. L'État requis exécute la demande d'entraide judiciaire aussi promptement que possible et tient compte dans toute la mesure possible de tous délais suggérés par l'État requérant et qui sont motivés, de préférence dans la demande. L'État requis répond aux demandes raisonnables de l'État requérant concernant les progrès réalisés dans l'exécution de la demande. Quand l'entraide demandée n'est plus nécessaire, l'État requérant en informe promptement l'État requis.

8. L'entraide judiciaire peut être refusée:

a Si la demande n'est pas faite conformément aux dispositions du présent projet d'annexe;

b Si l'État requis estime que l'exécution de la demande est susceptible de porter atteinte à sa souveraineté, à sa sécurité, à son ordre public ou à d'autres intérêts essentiels;

c Au cas où le droit interne de l'État requis interdirait à ses autorités de prendre les mesures demandées s'il s'agissait d'une infraction analogue ayant fait 
l'objet d'une enquête, de poursuites ou d'une procédure judiciaire dans le cadre de sa propre compétence;

d Au cas où il serait contraire au système juridique de l'État requis concernant l'entraide judiciaire d'accepter la demande.

9. Tout refus d'entraide judiciaire doit être motivé.

10. L'entraide judiciaire peut être différée par l'État requis au motif qu'elle entraverait une enquête, des poursuites ou une procédure judiciaire en cours.

11. Avant de refuser une demande en vertu du paragraphe $8 \mathrm{du}$ présent projet d'annexe ou d'en différer l'exécution en vertu du paragraphe 10, l'État requis étudie avec l'État requérant la possibilité d'accorder l'entraide sous réserve des conditions qu'il juge nécessaires. Si l'État requérant accepte l'entraide sous réserve de ces conditions, il se conforme à ces dernières.

12. L'État requis:

a Fournit à l'État requérant copie des dossiers, documents ou renseignements administratifs en sa possession et auxquels, en vertu de son droit interne, le public a accès; et

b Peut, à son gré, fournir à l'État requérant, intégralement, en partie ou aux conditions qu'il estime appropriées, copie de tous dossiers, documents ou renseignements administratifs en sa possession et auxquels, en vertu de son droit interne, le public n'a pas accès.

\section{Utilisation de l'information par l'État requérant}

13. L'État requérant ne communique ni n'utilise les informations ou les éléments de preuve fournis par l'État requis pour des enquêtes, poursuites ou procédures judiciaires autres que celles visées dans la demande sans le consentement préalable de l'État requis. Rien dans le présent paragraphe n'empêche l'État requérant de révéler, lors de la procédure, des informations ou des éléments de preuve à décharge. Dans ce cas, l'État requérant avise l'État requis avant la révélation et, s'il lui en est fait la demande, consulte ce dernier. Si, dans un cas exceptionnel, une notification préalable n'est pas possible, l'État requérant informe sans retard l'État requis de la révélation.

14. L'État requérant peut exiger que l'État requis garde le secret sur la demande et sa teneur, sauf dans la mesure nécessaire pour l'exécuter. Si l'État requis ne peut satisfaire à cette exigence, il en informe sans délai l'État requérant.

\section{Déposition de personnes provenant de l'État requis}

15. Sans préjudice de l'application du paragraphe 19 du présent projet d'annexe, un témoin, un expert ou une autre personne qui, à la demande de l'État requérant, consent à déposer au cours d'une procédure ou à collaborer à une enquête, à des poursuites ou à une procédure judiciaire sur le territoire relevant de la juridiction de l'État requérant ne sera pas poursuivi, détenu, puni ni soumis à d'autres restrictions de sa liberté personnelle sur ce territoire à raison d'actes, d'omissions ou de condamnations antérieurs à son départ du territoire de l'État requis. Cette immunité cesse lorsque le témoin, l'expert ou ladite personne ayant eu, pendant 
une période de quinze jours consécutifs ou toute autre période convenue par les États à compter de la date à laquelle ils ont été officiellement informés que leur présence n'était plus requise par les autorités judiciaires, la possibilité de quitter le territoire relevant de la juridiction de l'État requérant, y sont néanmoins demeurés volontairement ou, l'ayant quitté, y sont revenus de leur plein gré.

16. Lorsque cela est possible et conforme aux principes fondamentaux du droit interne, si une personne qui se trouve sur le territoire relevant de la juridiction d'un État doit être entendue comme témoin ou comme expert par les autorités judiciaires d'un autre État, le premier État peut, à la demande de l'autre, autoriser son audition par vidéoconférence s'il n'est pas possible ou souhaitable qu'elle comparaisse en personne sur le territoire relevant de la juridiction de l'État requérant. Les États peuvent convenir que l'audition sera conduite par une autorité judiciaire de l'État requérant et qu'une autorité judiciaire de l'État requis y assistera.

Transfert de personnes détenues dans l'État requis à des fins de témoignage

17. Toute personne détenue ou purgeant une peine sur le territoire relevant de la juridiction d'un État, dont la présence est requise dans un autre État à des fins d'identification ou de témoignage ou pour qu'elle apporte de toute autre manière son concours à l'obtention de preuves dans le cadre d'enquêtes, de poursuites ou de procédures judiciaires relatives aux infractions couvertes par les présents projets d'article, peut faire l'objet d'un transfèrement si les conditions ci-après sont réunies:

a Ladite personne y consent librement et en toute connaissance de cause; et

b Les autorités compétentes des deux États concernés y consentent, sous réserve des conditions que ces États peuvent juger appropriées.

18. Aux fins du paragraphe 17 du présent projet d'annexe:

a L'État vers lequel la personne est transférée a le pouvoir et l'obligation de la garder en détention, sauf demande ou autorisation contraire de la part de l'État à partir duquel elle a été transférée;

b L'État vers lequel la personne est transférée s'acquitte sans retard de l'obligation de la remettre à la garde de l'État à partir duquel elle a été transférée, conformément à ce qui aura été convenu au préalable ou autrement décidé par les autorités compétentes des deux États;

c L'État vers lequel la personne est transférée ne peut exiger de l'État à partir duquel elle a été transférée qu'il engage une procédure d'extradition pour qu'elle lui soit remise; et

d Il est tenu compte de la période que la personne a passée en détention dans l'État vers lequel elle a été transférée aux fins du décompte de la peine à purger dans l'État à partir duquel elle a été transférée.

19. À moins que l'État à partir duquel une personne doit être transférée en vertu des paragraphes 17 et 18 du présent projet d'annexe ne donne son accord, ladite personne, quelle que soit sa nationalité, n'est pas poursuivie, détenue, punie ni soumise à d'autres restrictions de sa liberté personnelle sur le territoire relevant 
de la juridiction de l'État vers lequel elle est transférée à raison d'actes, d'omissions ou de condamnations antérieurs à son départ du territoire relevant de la juridiction de l'État à partir duquel elle a été transférée.

Frais

20. Les frais ordinaires encourus pour exécuter une demande sont à la charge de l'État requis, à moins qu'il n'en soit convenu autrement entre les États concernés. Lorsque des dépenses importantes ou extraordinaires sont ou se révèlent ultérieurement nécessaires pour exécuter la demande, les États se consultent pour fixer les conditions selon lesquelles la demande sera exécutée, ainsi que la manière dont les frais seront assumés. 